\title{
Impact of Trypanosoma cruzi clonal evolution on its biological properties in mice
}

\author{
M.J. de O. Toledo, ${ }^{\mathrm{a}, \mathrm{e}}$ M. de Lana, ${ }^{\mathrm{b}, *}$ C.M. Carneiro, ${ }^{\mathrm{b}}$ M.T. Bahia, ${ }^{\mathrm{c}}$ \\ G.L.L. Machado-Coelho, ${ }^{\mathrm{d}}$ V.M. Veloso,${ }^{\mathrm{c}}$ C. Barnabé ${ }^{\mathrm{f}}$ M. Tibayrenc, ${ }^{\mathrm{f}}$ and W.L. Tafuri ${ }^{\mathrm{c}, \mathrm{e}}$ \\ a Departamento de Análises Clínicas, Bloco 190, Universidade Estadual de Maringá, Av. Colombo, 5790, 87020-900, \\ Maringá, PR, Brazil

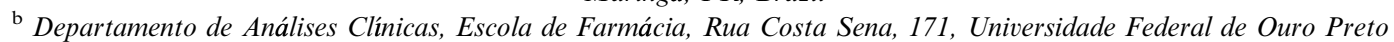 \\ (UFOP), 35400-000, Ouro Preto, MG, Brazil \\ ${ }^{\mathrm{c}}$ Departamento de Ciências Biológicas, Instituto de Ciências Exatas e Biológicas, Campus Universitário Morro do Cruzeiro, UFOP, \\ 35400-000, Ouro Preto, MG, Brazil \\ d Departamento de Farmácia, Escola de Farmácia, Rua Costa Sena, 171, UFOP, 35400-000, Ouro Preto, MG, Brazil \\ e Departamento de Parasitologia, Instituto de Ciências Biológicas, Universidade Federal de Minas Gerais, Av. Antônio Carlos, 6627, \\ Campus Pampulha, 31270-901, Belo Horizonte, MG, Brazil \\ ${ }^{\mathrm{f}}$ Génétique des Maladies Infectieuses, Unité Mixte de Recherche No. 9926 Centre National de la Recherche Scientifique (CNRS)/Institut de \\ Recherche pour le Développement (IRD), BP 5045, 34032, Montpellier Cedex 1, France
}

Received 7 June 2001; accepted 6 December 2001

\begin{abstract}
Twenty Trypanosoma cruzi stocks attributed to the 19, 20,39, and 32 clonal genotypes were comparatively studied in BALB/c mice during the acute and chronic phases of the infection to test the working hypothesis that $T$. cruzi clonal structure has a major impact on its biological properties. Fourteen parameters were assayed: (1) infectivity; (2) prepatent period; (3) patent period; (4) maximum of parasitemia; (5) day of maximum of parasitemia; (6) parasitemia; (7) mortality, (8) percentage of positive hemoculture, (9) tissue parasitism; (10) inflammatory process during the acute phase of the infection; (11) mortality, (12) percentage of positive hemoculture; (13) tissue parasitism; and (14) inflammatory process during the chronic phase of the infection. Statistical comparison showed that the results are overall consistent with the working hypothesis that biological differences are proportional to the evolutionary divergence among the genotypes. Thus, closely related genotypes (19 vs 20 and 32 vs 39) show in general fewer differences than distantly related groups (19 or 20 vs 32 or 39) except for the comparison between 19 and 32 . The working hypothesis is even more strongly supported by the result of the nonparametric Mantel test, which showed a highly significant correlation $\left(P=2.3 \times 10^{-3}\right)$ between biological differences and genetic distances among all pairs of stocks. These data taken together emphasize that it is crucial to take into account the phylogenetic diversity of $T$. cruzi natural clones in all applied studies dealing with diagnosis, drug and vaccine design, epidemiological surveys, and clinical diversity of Chagas' disease.
\end{abstract}

Index Descriptors and Abbreviations: Trypanosoma cruzi; phylogenetic distance; biological properties; clonal theory; multilocus enzyme electrophoresis (MLEE); randomly amplified polymorphic DNA (RAPD); acute phase (AP); chronic phase (CP); days after inoculation (d.a.i.); liver infusion tryptose (LIT); gastrointestinal tract (GIT); genitourinary tract (GUT); percentage of infectivity (\%INF); percentage of mortality during the acute phase (\%MORT AP); percentage of mortality during the chronic phase (\%MORT $\mathrm{CP}$ ); prepatent period (PPP); patent period (PP); maximum of parasitemia (MP); day of maximum of parasitemia (DMP); parasitemia (PAR); percentage of positive hemoculture during the acute phase $(\%+\mathrm{HC}$ AP); percentage of positive hemoculture during the chronic acute phase $(\%+\mathrm{HC} \mathrm{CP})$; tissue parasitism (TP); inflammatory process (IP); tissue parasitism during the acute phase (TP AP); tissue parasitism during chronic phase (TP CP); inflammatory process during acute phase (IP AP); inflammatory process chronic phase (IP CP); Mann-Whitney test (MW); Kruskal-Wallis (KW); Kolmogorow-Smirnov test (KS). ( 2002 Elsevier Science (USA). All rights reserved.

\footnotetext{
${ }^{*}$ Corresponding author. Fax: +55-21-31-3-559-16-28.

E-mail address: delana@cpd.ufop.br (M. de Lana).
} 


\section{Introduction}

Trypanosoma cruzi, the aetiological agent of Chagas' disease, exhibits a great biological diversity for many parameters (Andrade, 1976; Andrade et al., 1985; Dvorak, 1984; Melo and Brener, 1978). Moreover, the clinical manifestations of the disease are also diverse (Brener, 1987; Dias, 1992; Rassi and Luquetti, 1992). Miles et al. (1981b) have suggested that this variability could be in part explained by this parasite's genetic diversity. Several authors have indeed shown an association between $T$. cruzi isoenzyme variability and its biological properties (Andrade and Magalhães, 1997; Andrade et al., 1992, 1983; Carneiro et al., 1991; Dvorak et al., 1980; Marques De Araújo and Chiari, 1988). However, these studies lacked either a sufficient sample size or a rigorous population genetic framework or both.

Tibayrenc et al. (1986) and Tibayrenc and Ayala (1988) have proposed that T. cruzi undergoes predominant clonal evolution with only rare events of genetic recombination. This clonal model predicts a parallel evolution between biological differences and genetic divergence among $T$. cruzi natural clones. Such a statistical association between genetic and biological differences has been found experimentally, in vitro by Laurent et al. (1997) and Revollo et al. (1998), and in the vector by Lana et al. (1998).

The present work explores the same working hypothesis in experimental infection in BALB/c mice.

\section{Materials and methods}

\subsection{Parasites}

The same standardized sample of $20 T$. cruzi stocks used for previous studies (Lana et al., 1998; Laurent et al., 1997; Revollo et al., 1998) has been used. The stocks were cloned by dilution and micromanipulation, with visual verification under microscope. They were fully characterized by MLEE with 15 "loci" (Tibayrenc and Ayala, 1988), then with both MLEE with 22 different loci (Barnabé et al., 2000) and RAPD with 10 primers (Tibayrenc et al., 1993). They are representative of the "major clonets" (widespread clonal genotypes) 19, 20, 32, and 39 (Tibayrenc and Ayala, 1988). According to the nomenclature recently proposed (Satellite Meeting, Rio de Janeiro, Anon, 1999), major clonets 19 and 20 are included in T. cruzi I (Zymodeme 1 of Miles et al., 1981a; Lineage 1 of Tibayrenc, 1995; and lineage 2 of Souto et al., 1996) whereas both 32 and 39 groups are included in T. cruzi II (Zymodeme 2 of Miles et al., 1981a; Lineage 2 of Tibayrenc, 1995; and lineage 1 of Souto et al., 1996. Clonet 39 is equivalent to lineage $1 / 2$ of Souto et al. (1996) considered as a hybrid clonal genotype (Brisse et al., 1998, 2000; Machado and Ayala, 2001). Information on the laboratory code, host, and geographic origin of these stocks is given in Table 1. The clonal genotypes 19, 20, 39, and 32 illustrate different phylogenetic relationships. Genotypes 32 and 39 are more closely related to each other, whereas genotype 19/

Table 1

Laboratory code, host and geographic origin of the 20 Trypanosoma cruzi stocks used in the present study

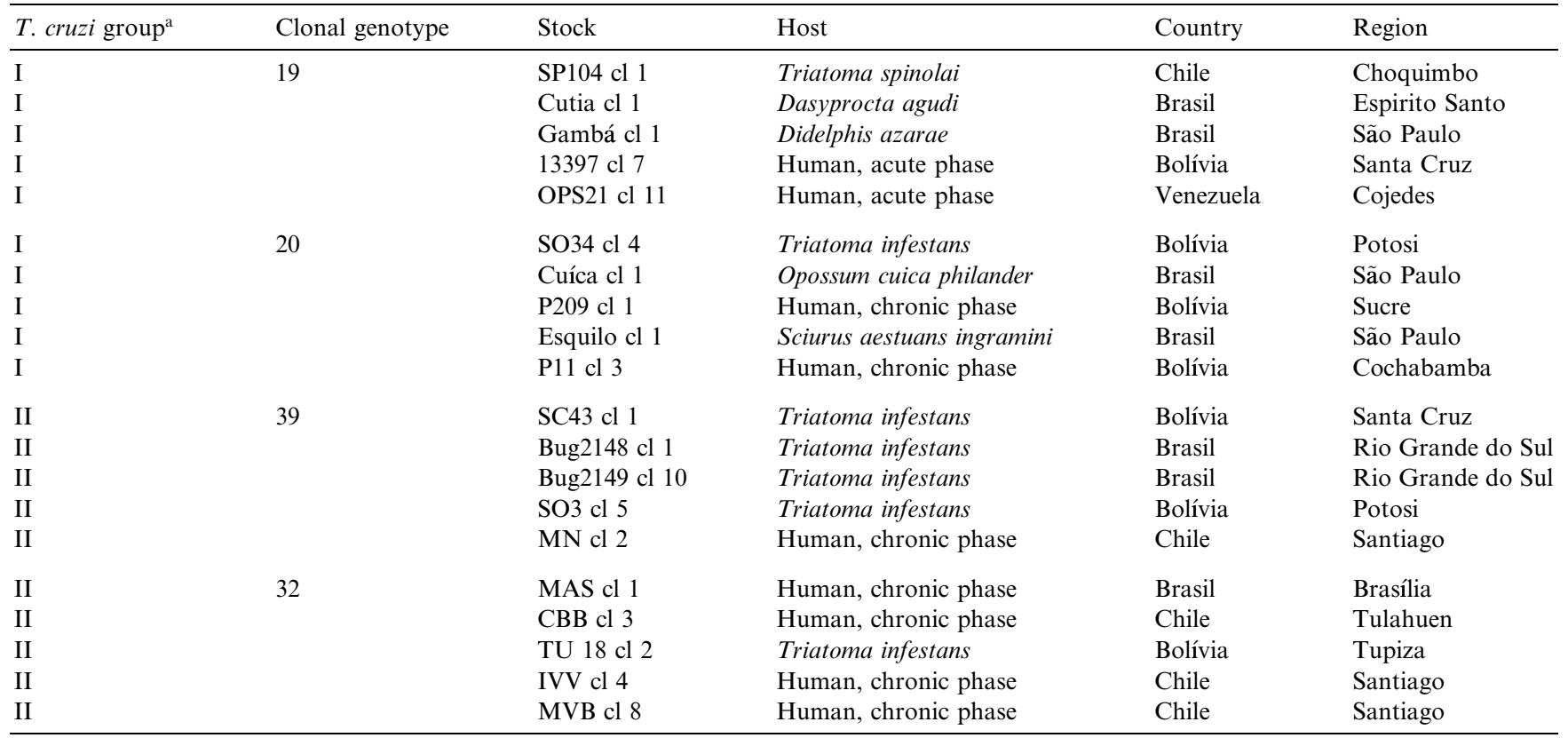

${ }^{a}$ Recommendations from Anon (1999). 
20 is more distantly related to both 32 and 39 (Fig. 1). Lastly, 19 and 20 are very close to each other.

\subsection{Experimental conditions}

Groups of 20 female BALB/c mice originating from the Instituto de Ciências Biológicas-UFMG, 28-30 days old, were inoculated through intraperitoneal route with 10,000 blood trypomastigotes/animal of each stock studied. Inocula were obtained from breast-fed Swiss mice in which the stocks were previously inoculated with a high number of metacyclic trypomastigotes obtained from late stationary culture in LIT medium after treatment with guinea pig serum according to Deane et al. (1984).

During the AP of the infection, mice were checked every day for PAR and mortality registration until 90 d.a.i. Deaths that occurred between 90 days and 12 months after inoculation were registered as mortality during the CP. Inocula and PAR were counted according to Brener (1962). Sixty and 140 d.a.i. hemoculture in LIT medium (Filardi and Brener, 1987) was made with

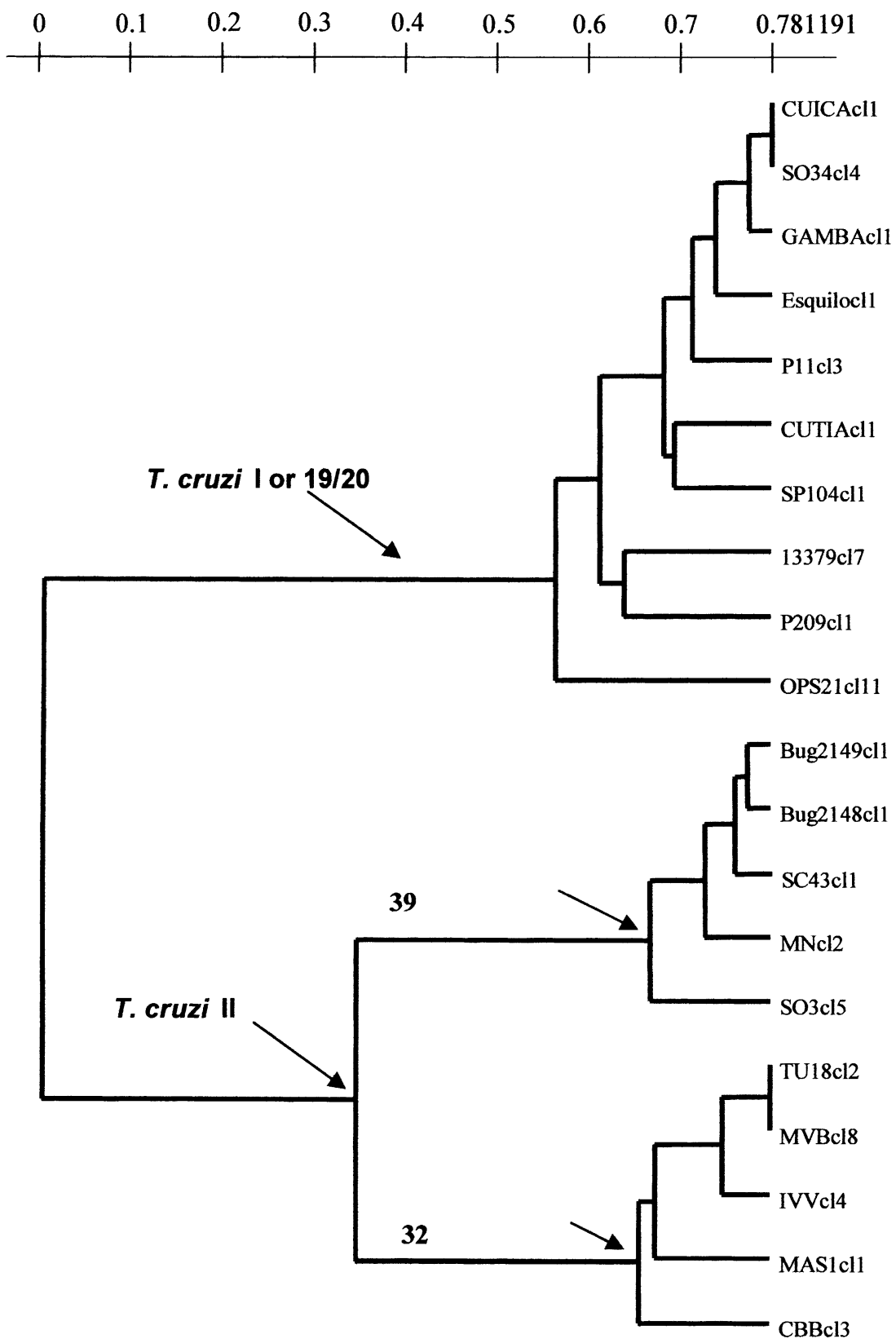

Fig. 1. An unweighted pair-group method with arithmetic averages (UPGMA) dendrogram depicting the phylogenetic relationships among the 20 Trypanosoma cruzi stocks under study, assayed by 22 isoenzyme loci (Tibayrenc et al., 1993). Top cluster corresponds to clonal genotype 19/20 (T. cruzi I), medium cluster corresponds to clonal genotype 39 (T. cruzi II), and bottom cluster corresponds to clonal genotype 32 (T. cruzi II). The scale indicates genetic distances estimated with the index of Jaccard (1908). 
approximately $400 \mu \mathrm{L}$ of blood taken from each mouse (10 for AP and 10 for $\mathrm{CP}$ ) through the retro-orbital plexus vein. Afterwards, four of these mice, two in the 30th d.a.i (AP) and two in the 110th d.a.i. (CP), were necropsied for histopathological studies. The following organs and tissues were collected: (1) heart, (2) skeletal muscle, (3) GIT including stomach, small and large intestine, (4) GUT including bladder, kidney and uterine tubes, (5) brain, (6) liver, (7) pancreas, and (8) fat tissue. This material was routine processed, included in paraffin. Sections $5 \mu \mathrm{m}$ thick of three blocks containing all organs of each mouse were obtained for further histopathological analysis. These preparations were stained with hematoxylin-eosin for microscope examinations.

In some cases (animals infected with the SO34c14, $\mathrm{P} 209 \mathrm{c} 11$ and $\mathrm{MNc} 12)$ the immunohistochemical peroxidase anti-peroxidase preparation (Barbosa, 1985) was processed to increase the detection of amastigotes.

\subsection{Parameters evaluated}

(a) \%INF: percentage of mice with positive fresh blood examination and/or hemoculture.

(b) Mortality expressed in cumulative percentage of death registered during the AP (\%MORT AP) and during the $\mathrm{CP}$ of infection (\%MORT CP).

(c) PPP: the first day with positive fresh blood examination.

(d) PP: the period with positive fresh blood examination.

(e) MP expressed in the number of trypomastigotes/ $0.1 \mathrm{~mL}$ of blood, detected daily in fresh blood examination.

(f) DMP.

(g) PAR: area under the curve.

(h) Percentage of mice with positive hemoculture during the acute $(\%+\mathrm{HC}$ AP- 60 th d.a.i) and chronic phases $(\%+\mathrm{HC}$ CP-140 d.a.i) of the infection.

(i) TP: number of organs or tissues with parasitism classified as absent $(-)$, mild $(+)$, moderate $(++)$, and severe $(+++)$ during the acute (TP AP) and chronic (TP $\mathrm{CP})$ phases of the infection.

(j) IP: number of organs or tissues with inflammatory process classified as absent $(-)$, mild $(+)$, moderate $(++)$, and severe (+++) during the acute (IP AP) and chronic phases (IP CP) of the infection.

\subsection{Statistical analysis}

The statistical analysis of the parameters of virulence was done as follows: PPP, PP, MP, and DMP were compared with the nonparametric MW (Conares, 1980). Parameters \%INF, \%MORT, and \%+HC were compared by chi-square test $\left(\chi^{2}\right)$. The area under the curve of PAR was compared with the nonparametric KS. The histopathologic data (TP and IP) were analyzed with the nonparametric KW (Snedecor and Cochran, 1989).

A global analysis of the data was done with the nonparametric Mantel test (Mantel, 1967), which makes it possible to evaluate a possible correlation between, on one hand, genetic distances measured by either MLEE or RAPD, on the other hand, biological differences among the stocks. Contrary to the classical correlation test, this randomization procedure does not need any assumptions about the number of degrees of freedom. This procedure gives an equal weight to each biological parameter in the overall biological distance.

\section{Results}

\subsection{Parameters of virulence}

A great standard deviation was observed in PPP, PP, MP, and DMP showing that clones of the same genetic group are not homogeneous in their characteristics (Table 2). The mortality parameter was similar for the different genetic groups during the AP and significantly different only between the genotypes 20 and 39 during the $\mathrm{CP}$ of the infection. The rates of mortality during the $\mathrm{CP}$ were significantly higher $(p<0.001)$ than those observed during the AP of the infection (Table 3).

\subsubsection{Infection with T. cruzi I (clonal genotypes 19 and 20)}

Significant differences in four out of 14 parameters (Table 3) were observed in mice infected with the genetic groups 19 and 20, phylogenetically closely related, both

Table 2

Mean values and standard deviations of biological parameters under study in mice infected with Trypanosoma cruzi of the genetic groups 19, 20, 39, and 32

\begin{tabular}{|c|c|c|c|c|c|}
\hline \multirow[t]{2}{*}{ Parameter } & \multirow[t]{2}{*}{ Abbreviation } & \multicolumn{4}{|c|}{ Genetic groups } \\
\hline & & 19 & 20 & 39 & 32 \\
\hline Prepatent period (days) & PPP & $14.4 \pm 6.5$ & $11.3 \pm 5.5$ & $11.1 \pm 6.2$ & $13.6 \pm 5.5$ \\
\hline${ }^{\mathrm{a}}$ Maximum of parasitemia $\left(\times 10^{3}\right)$ & MP & $19 \pm 25$ & $297 \pm 550$ & $107 \pm 186$ & $18 \pm 11$ \\
\hline Day of MP (days) & DMP & $26.4 \pm 13.5$ & $27.0 \pm 8.0$ & $21.8 \pm 11.1$ & $26.1 \pm 12.2$ \\
\hline
\end{tabular}

${ }^{\mathrm{a}}$ Number of trypomastigotes $/ 0.1 \mathrm{~mL}$ of blood. 
Table 3

Comparison of fourteen parameters under study in mice infected with Trypanosoma cruzi of the genetic groups 19, 20, 39, and 32

\begin{tabular}{|c|c|c|c|c|c|c|c|}
\hline Parameter & $\begin{array}{l}\text { Statistic } \\
\text { method }\end{array}$ & $19 \times 20\left(n_{19}, n_{20}\right)$ & $19 \times 39\left(n_{19}, n_{39}\right)$ & $19 \times 32\left(n_{19}, n_{32}\right)$ & $20 \times 39\left(n_{20}, n_{39}\right)$ & $20 \times 32\left(n_{20}, n_{32}\right)$ & $39 \times 32\left(n_{39}, n_{32}\right)$ \\
\hline Prepatent period & MW & $156.3 \times 107.6^{*}(110,146)$ & $133.8 \times 88.41^{*}(110,111)$ & $81.7 \times 76.2^{* * *}(110,49)$ & $130.4 \times 127.1^{* * *}(146,111)$ & $90.5 \times 120.4^{*}(146,49)$ & $72.2 \times 99.3^{*}(111,49)$ \\
\hline Patent period & MW & $74.0 \times 88.2^{* * *}(71,92)$ & $72.9 \times 63.8^{* * *}(71,65)$ & $54.0 \times 52.4^{* * *}(71,35)$ & $88.1 \times 66.2^{*}(92,65)$ & $70.6 \times 46.6^{*}(92,35)$ & $49.3 \times 52.7^{* * *}(65,35)$ \\
\hline Maximum of parasitemia & MW & $48.2 \times 100.0^{*}(70,81)$ & $61.0 \times 74.6^{* *}(70,64)$ & $48.8 \times 61.3^{* *}(70,35)$ & $86.7 \times 55.7^{*}(81,64)$ & $69.9 \times 32.2^{*}(81,35)$ & $50.0 \times 50.0^{* * *}(64,35)$ \\
\hline $\begin{array}{l}\text { Day of maximum of para- } \\
\text { sitemia }\end{array}$ & MW & $71.9 \times 83.6^{* * *}(74,81)$ & $74.9 \times 63.3^{* * *}(74,64)$ & $54.7 \times 55.6^{* * *}(74,35)$ & $81.9 \times 61.7^{*}(81,64)$ & $60.3 \times 54.3^{* * *}(81,35)$ & $46.9 \times 55.8^{* * *}(64,35)$ \\
\hline $\begin{array}{l}\text { Tissue parasitism acute } \\
\text { phase }\end{array}$ & $\mathrm{KW}$ & $38.9 \times 42.1^{* * *}(40,40)$ & $42.5 \times 38.5^{* *}(40,40)$ & $41.5 \times 39.5^{* * *}(40,40)$ & $44.0 \times 37.0^{* *}(40,40)$ & $43.1 \times 39.9^{* * *}(40,40)$ & $39.5 \times 41.5^{* * *}(40,40)$ \\
\hline $\begin{array}{l}\text { Tissue parasitism chronic } \\
\text { phase }\end{array}$ & KW & $40.5 \times 40.5^{* * *}(40,40)$ & $38.5 \times 42.5^{* *}(40,40)$ & $40.5 \times 40.5^{* * *}(40,40)$ & $38.5 \times 42.5^{* *}(40,40)$ & $40.5 \times 40.5^{* * *}(40,40)$ & $42.5 \times 38.5^{* *}(40,40)$ \\
\hline $\begin{array}{l}\text { Inflammatory process acute } \\
\text { phase }\end{array}$ & $\mathrm{KW}$ & $37.6 \times 43.6^{* * *}(40,40)$ & $42.4 \times 38.6^{* * *}(40,40)$ & $39.2 \times 41.9^{* * *}(40,40)$ & $45.5 \times 35.5^{* *}(40,40)$ & $42.5 \times 38.6^{* * *}(40,40)$ & $36.8 \times 44.2^{* * *}(40,40)$ \\
\hline $\begin{array}{l}\text { Inflammatory process } \\
\text { chronic phase }\end{array}$ & $\mathrm{KW}$ & $42.7 \times 38.4^{* * *}(40,40)$ & $46.8 \times 34.2^{*}(40,40)$ & $42.1 \times 39.0^{* * *}(40,40)$ & $44.3 \times 36.7^{* * *}(40,40)$ & $39.5 \times 41.6^{* * *}(40,40)$ & $34.7 \times 46.3^{* *}(40,40)$ \\
\hline Infectivity & $\chi^{2}$ & $91.4 \times 98.3^{*}(301,330)$ & $91.3 \times 70.3^{*}(301,311)$ & $91.3 \times 67.1^{*}(301,296)$ & $98.3 \times 70.3^{*}(330,311)$ & $98.3 \times 67.1^{*}(330,296)$ & $703 \times 67.1^{* * *}(311,296)$ \\
\hline Mortality acute phase & $\chi^{2}$ & $1.9 \times 5.4^{* * *}(132,153)$ & $1.9 \times 2.9^{* * *}(132,136)$ & $1.9 \times 2.9^{* * *}(132,136)$ & $5.4 \times 2.9^{* * *}(153,136)$ & $5.4 \times 2.9^{* * *}(153,136)$ & $2.9 \times 2.9^{* * *}(136,136)$ \\
\hline Mortality chronic phase & $\chi^{2}$ & $22.4 \times 18.8^{* * *}(132,153)$ & $22.4 \times 31.9^{* * *}(132,136)$ & $22.4 \times 18.3^{* * *}(132,136)$ & $18.3 \times 31.9^{* *}(153,136)$ & $18.8 \times 18.3^{* * *}(153,136)$ & $31.9 \times 18.3^{* * *}(136,136)$ \\
\hline Hemoculture acute phase & $\chi^{2}$ & $89.3 \times 95.5^{* * *}(49,44)$ & $89.3 \times 57.6^{*}(49,48)$ & $89.3 \times 82.8^{* * *}(49,49)$ & $95.5 \times 57.6^{*}(44,48)$ & $95.5 \times 82.8^{* * *}(44,49)$ & $57.6 \times 82.8^{*}(48,49)$ \\
\hline Hemoculture chronic phase & $\chi^{2}$ & $100.0 \times 98.0^{* * *}(49,58)$ & $100.0 \times 40.9^{*}(49,56)$ & $100.0 \times 81.0^{*}(49,51)$ & $98.0 \times 40.9^{*}(58,46)$ & $98.0 \times 81.0^{*}(58,51)$ & $40.9 \times 81.0^{*}(46,51)$ \\
\hline Parasitemia & KS & $11.07^{*}(109,96)$ & $3.99^{*}(109,107)$ & $4.53^{*}(109,97)$ & $11.06^{*}(96,107)$ & $15.37^{*}(96,97)$ & $5.16^{*}(107,97)$ \\
\hline No of significant differences & & 4 & 9 & 4 & 11 & 6 & 6 \\
\hline
\end{tabular}

Mean rank (MW = Mann-Whitney test, KW = Kruskal-Wallis test); Percentual ( $\chi^{2}=$ chi-square test); $Z$ of Kolmogorov-Smirnov (KS $=$ Two-Sample Kolmogorov-Smirnov test). ${ }^{*} P<0.005$.

${ }^{* *} P<0.05$.

${ }^{* * *}$ Not significant. 
T. cruzi I. The genetic group 19 with lower PAR and MP showed a higher value of PPP than genetic group 20 (Tables 3 and 4, Fig. 2). Clones of the genetic group 20 were significantly more infective and they led to a higher PAR (fresh blood examination) during the AP of the infection than clones from the genotype 19 (Table 3).

All five cloned stocks of this genotype induced patent PAR in mice while genotype 19 includes one subpatent stock (SP104 cl1).

\subsubsection{Infection with T. cruzi II (clonal genotypes 39 and 32)}

Mice infected with the stocks pertaining to the clonal genotypes 39 and 32 closely related both $T$ cruzi II and phylogenetically distant from the genotypes 19 and 20, displayed significant differences in 6 out of 14 of the same parameters studied (Table 3). Mice infected with the genetic group 39 with a higher PAR (Fig. 2) showed the lowest values of PPP and percentages of positive hemocultures (in both phases of infection) (Table 2). Mice infected with the five stocks of the genetic group 39 showed the most dispersed results among them in relation to many variables (individual data not shown). This group displayed the second highest MP and PAR (Fig. 2), mostly due to the high values of the stock Bug2149 c110 for these variables.

Only three stocks from the genotype 39 and two from the genotype 32 showed patent PAR in mice.

\subsection{Histopathological data}

The histopathological results revealed in general the presence of few amastigotes and inflammatory lesions. Parasitism was observed only in 5 organs/tissue exam- ined: GIT and GUT were both more involved in this process, followed by skeletal muscles, heart and fat tissue. Inflammatory process was detected in all organs/ tissue examined except in the brain, and in general, the organs and tissues more affected were also GUT/GIT, skeletal musculature and heart.

Out of the 20 stocks studied, only nine $(45 \%)$ displayed a visible TP, generally classified as mild. Parasitism was observed for three stocks of genotype 20 (AP), two stocks of genotype 19 (AP), three stocks of genotype 39 (CP), and one stock of genotype 32 (AP). Parasitism was observed in $8.13 \%$ of the organs/tissue in the AP (30th d.a.i.), and in $2.5 \%$ of the organs/tissue during the CP (110th d.a.i.) The number of organs/tissue with parasitism in 40 analyzed by genotype during the AP was 4 , 7, 0 , and 2 for the genotypes 19, 20,39, and 32, respectively (Table 4). Only mice infected with stocks from genotype 39 showed parasitism (four out of 40 organs/ tissue analyzed) during the CP (Table 4). Immunohistochemical analysis did not change these results.

Inflammatory process was present in mice infected with all 20 stocks. Most cases were classified as mild, whereas severe inflammatory reactions were less. The inflammatory process was observed in $51.30 \%$ of the organs/tissue in the AP (30th d.a.i.), and in $45.63 \%$ of the organs/tissue during the $\mathrm{CP}$ (110th d.a.i). The numbers of organs/tissue with inflammatory process in 40 analyzed by genotype were: $18,24,16$, and 24 during the AP and 22, 17, 11, and 23 during the $\mathrm{CP}$ for the genotypes 19, 20, 39, and 32, respectively (Table 4).

The genotypes 19, 20, and 32 displayed parasitism only during the AP (Table 4, Figs. 3A, C, and D). The genotype 39 was the only to show parasitism in the $\mathrm{CP}$ (three out of the five stocks) (Table 4, Figs. 4A-C).

Table 4

Number of organs or tissues with parasitism and inflammatory process in mice infected with Trypanosoma cruzi of the genetic groups 19, 20, 39, and 32 during the acute and chronic phases of the infection

\begin{tabular}{|c|c|c|c|c|c|c|c|}
\hline \multirow[t]{2}{*}{ Parameter } & \multirow{2}{*}{$\begin{array}{l}\text { Phase of } \\
\text { infection }\end{array}$} & \multirow{2}{*}{$\begin{array}{l}\text { Number of } \\
\text { organs/tissue }\end{array}$} & \multirow[t]{2}{*}{ Intensity } & \multicolumn{4}{|c|}{ Genetic groups } \\
\hline & & & & 19 & 20 & 39 & 32 \\
\hline \multirow[t]{6}{*}{ Tissue parasitism } & Acute & 40 & - & 36 & 33 & 40 & 38 \\
\hline & & & + & 4 & 5 & 0 & 2 \\
\hline & & & ++ & 0 & 2 & 0 & 0 \\
\hline & Chronic & 40 & - & 40 & 40 & 36 & 40 \\
\hline & & & + & 0 & 0 & 1 & 0 \\
\hline & & & ++ & 0 & 0 & 3 & 0 \\
\hline \multirow[t]{7}{*}{ Inflammatory process } & Acute & 40 & - & 22 & 16 & 24 & 16 \\
\hline & & & + & 8 & 12 & 11 & 18 \\
\hline & & & ++ & 10 & 10 & 5 & 6 \\
\hline & & & +++ & 0 & 2 & 0 & 0 \\
\hline & Chronic & 40 & - & 18 & 23 & 29 & 17 \\
\hline & & & + & 12 & 8 & 9 & 20 \\
\hline & & & ++ & 10 & 9 & 2 & 3 \\
\hline
\end{tabular}

- (Absent); + (mild); ++ (moderate); +++ (severe). 


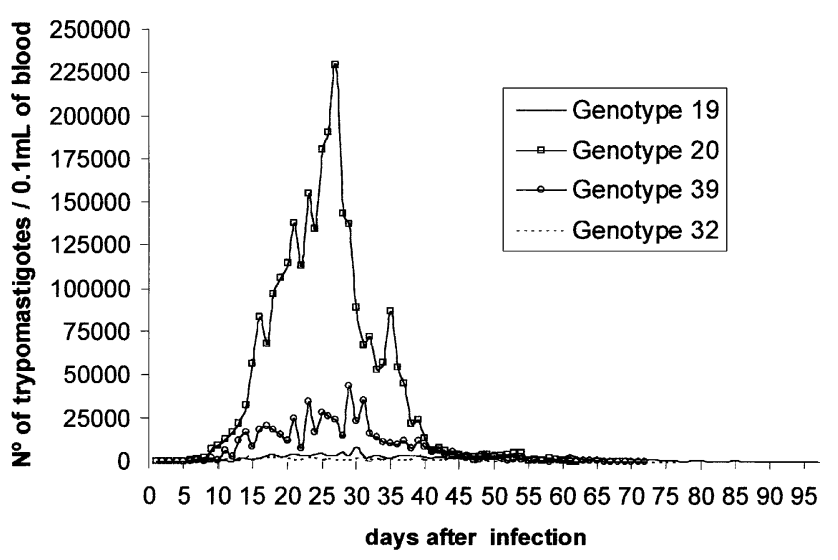

Fig. 2. Curve of mean parasitemia in mice infected with the clonal genotypes 19, 20, 39, and 32 of Trypanosoma cruzi.

\subsubsection{Infection with T.cruzi I (clonal genotypes 19 and 20)}

Histopathological results during both phases of the infection were not significantly different between animals infected with genotypes 19 and 20 (Table 3). However, genotype 20 showed more severe parasitism during the AP than the genotype 19 (Table 4 and Figs. $3 \mathrm{C}$ and D). During the $\mathrm{CP}$ parasites were not observed in mice infected with these genotypes.

During the AP of the infection severe inflammatory reactions were observed only in mice infected with the genotype 20 (Table 4 and Fig. 3B). Moderate inflammatory reaction (Figs. 3A, C, and D) was twice more frequent in mice infected with genotypes 19 and 20 in relation to the other genotypes (Table 4 ).

\subsubsection{Infection with T. cruzi II (clonal genotypes 39 and 32)}

Histopathological results were significantly different only during the $\mathrm{CP}$ of the infection for both the parasitism and inflammatory process between animals infected with the genotypes 39 and 32 (Table 3). Stocks of T. cruzi II showed during the AP of the infection only mild parasitism and only in mice infected with the genotype 32 (Table 4). Differently from the other three genotypes studied, only mice infected with stocks from the genotype 39 did not show parasitism during the AP of the infection. In contrast, this genotype was the only one that displayed parasitism during the $\mathrm{CP}$ of the infection (Table 4) especially in skeletal musculature and TGI. This was observed with three out of five stocks studied (Bug2149 c110, MN c12, and SO3 c15).

Mice infected with genotype 32 showed a higher number of organs/tissue with mild inflammatory reaction (Fig. 4D) in relation to animals of the other genotypes in both phases of the infection (Table 4). The lowest number of organs/tissue with inflammatory reaction among all genotypes was observed in mice infected with genotype 39 during the acute and also during the $\mathrm{CP}$ of the infection when only mice infected with this genotype showed TP (Table 4 and Figs. 4A-C).

\subsection{Statistical comparisons between $T$. cruzi $I$ and $T$. cruzi II genotypes}

Significant differences observed between the clonal genotypes 20 vs 39,19 vs 39,20 vs 32 , and 19 vs 32 were $11,9,6$, and 4, respectively (Table 3 ). In the comparison 20 vs 39 , genotype 20 showed higher values of PP, MP, DMP, \%INF, \%+HC (both phases of the infection), PAR, TP AP, and IP AP than genotype 39, except TP CP (Table 3).

When genetic groups 19 vs 39 were compared, genotype 19 showed higher values of PPP, $\% \mathrm{INF}, \%+\mathrm{HC}$ in both phases of the infection, TP AP and inflammatory process $(\mathrm{CP})$ than genotype 39 . On the other hand genotype 39 showed higher values of MP, PAR, and TP CP than genotype 19 (Table 3).

In the comparison of 20 vs 32 , genotype 20 showed higher values of PP, MP, \%INF, \% + HC (CP), and PAR than genotype 32, except PPP (Table 3).

Finally in the comparison of 19 vs 32, genotype 19 showed higher values of \%INF, \% + HC (CP), and PAR than genotype 32 (Table 3). The MP was higher in group 32.

Overall, the decreased order of the four genetic groups considering all the parameters here studied for the 20 stocks is $20>19>32>39$.

The result of the Mantel test evaluating the correlation between the matrices of 13 biological parameters (except PAR = area under the curve of PAR) and genetic distances for all pairs of stocks was significant $\left(P=2.3 \times 10^{-3}\right)$.

\section{Discussion}

Many authors have tried to find a link between the genetic diversity of T. cruzi and this parasite's biological diversity. The biological parameters tested were various in experimental conditions in vitro (Dvorak et al., 1980), behavior in vectors and in mice (Garcia and Dvorak, 1982;Andrade et al., 1983; Carneiro et al., 1991), and clinical manifestations of the disease in chagasic patients from which the parasite was isolated (Brenière et al., 1985; Miles et al., 1981b). Andrade and Magalhães (1997) studying 138 T. cruzi stocks isolated from various hosts and vast geographic areas of Lathi America classified the stocks in three groups called "biodemes" which were correlated with "zymodemes." Although the above-cited studies have brought significant contributions, none of them relied on a clear population genetic framework. The present work, as well as previous ones published by other researchers of the same team (Lana et al., 1998; Laurent et al., 1997; Revollo et al., 1998), 

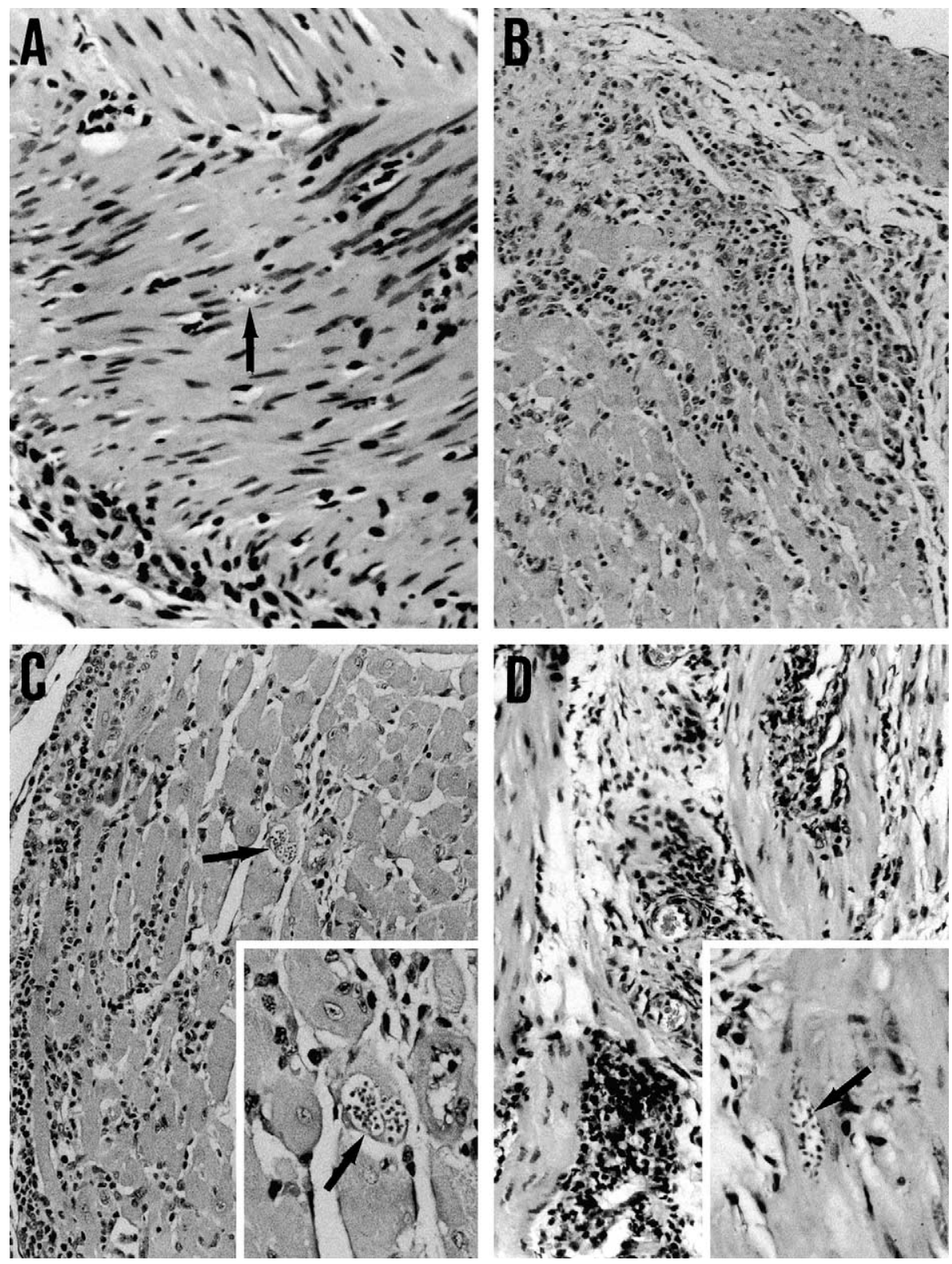

Fig. 3. Microphotograph of BALB/c mice infected with Trypanosoma cruzi I. (A) Genitourinary tract with tissue parasitism of SP104 cl1 stock, genotype 19, and focal moderate inflammatory process predominantly mononuclear during the AP, 30th day of infection (660 $\times$ HE). (B) Severe myocarditis with SO34 c14 stock, genotype 20 during the AP $(330 \times \mathrm{HE})$. (C) Moderate myocarditis and parasitism with SO34 c14 stock, genotype 20 $(330 \times \mathrm{HE})$. Insert with amastigote nest $(660 \times \mathrm{HE})$. (D) Genitourinary tract showing moderate inflammatory process during the chronic phase $(110$ th day of infection) with P11 cl3 stock, genotype $20(330 \times$ HE). Insert with amastigote nest during the acute phase of infection with P209 cl1 stock, genotype $20(660 \times \mathrm{HE})$. Arrows indicate amastigote nest.

aimed to fill up this gap. Based on the working hypothesis of $T$. cruzi clonal evolution (Tibayrenc et al., 1986), a standard set of 20 stocks representative of four "major clonets" (frequently isolated, ubiquitous clonal genotypes of major epidemiological relevance) as well as of the whole phylogenetic diversity of T. cruzi, was analyzed for 14 different biological parameters.

The analysis of the 14 biological properties studied (all parameters of virulence and pathogenicity in mice) in relation with the genetic diversity is in agreement with the working hypothesis (Tibayrenc and Brenière, 1988).
Our results are also consistent and corroborate previous observations in vitro and in BALB/c mice (Laurent et al., 1997), in acellular and cellular cultures (Revollo et al., 1998), and in Triatoma infestans (Lana et al., 1998) which confirmed the correlation between phylogenetic distance among $T$. cruzi clonal genotypes and their biological properties. Although our data of experimental infection in BALB/c mice show the same general pattern as the results of Laurent et al. (1997), they cannot be compared directly because our animals were not immunosuppressed. 


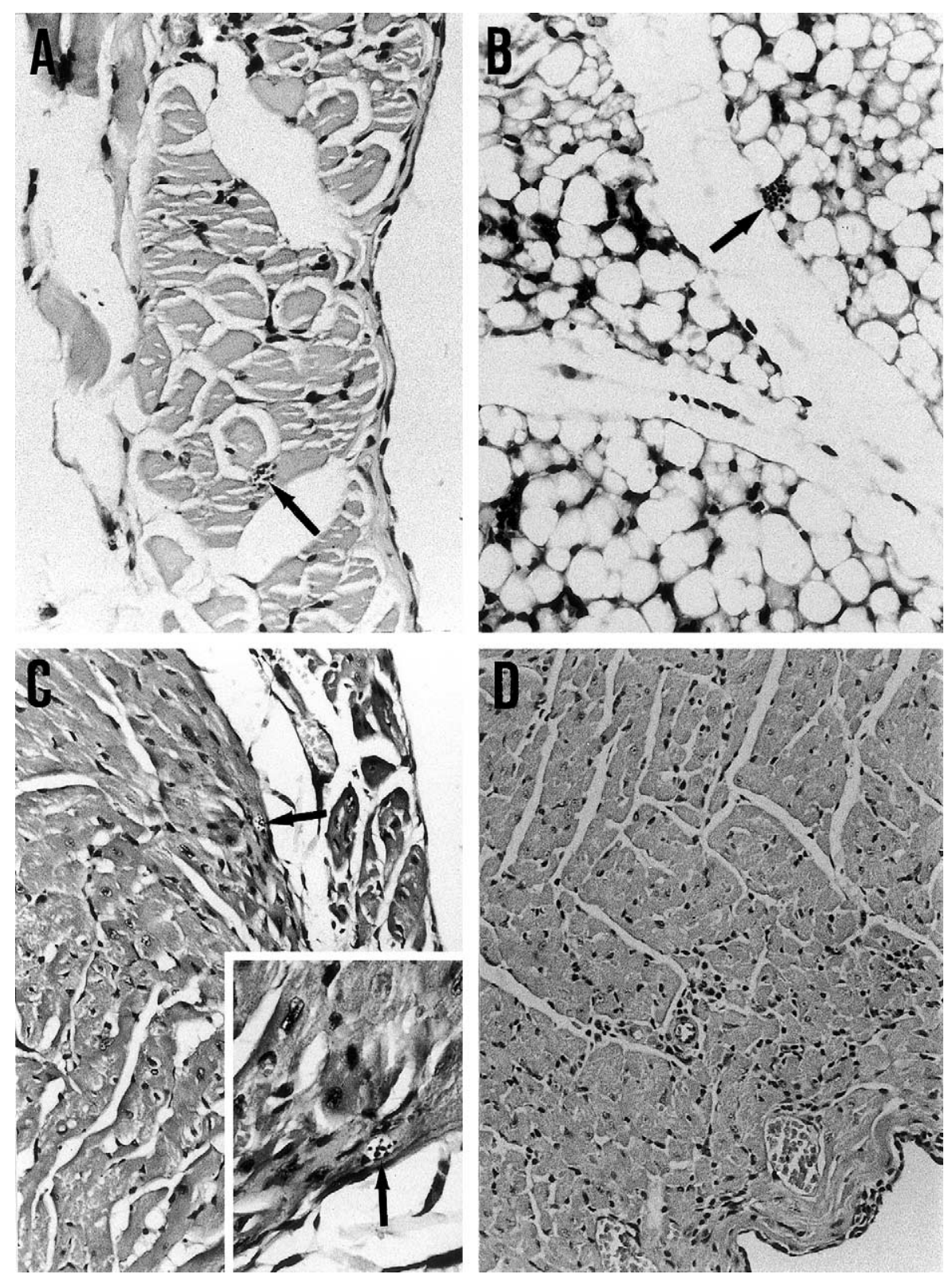

Fig. 4. Microphotograph of BALB/c mice infected with Trypanosoma cruzi II. (A) Skeletal muscle showing parasitism and mild inflammatory process during the chronic phase (110th day of infection) with MN cl2 stock, genotype 39 (660× HE). (B) Fat tissue showing parasitism and mild inflammatory process during the chronic phase with Bug2149 cl10 stock, genotype $39(660 \times \mathrm{HE})$. (C) Mild myocarditis and parasitism during the chronic phase of infection) with SO3 c15 stock, genotype 39 (330× HE). Insert showing amastigote nest (660× HE). (D) Mild myocarditis and pericarditis with mononuclear infiltrate during the acute phase (30th day of infection) with MAS cll stock, genotype 32 (330× HE). Arrows indicate amastigote nest.

As for these previous analyses, a great standard deviation in the majority of the parameters for a given clonet was observed again. Only the mortality parameter was similar for all genetic groups during AP although significant differences were observed between the genetic groups 20 and 39 during $\mathrm{CP}$ of the infection.

The great majority of clonal stocks studied were of low virulence. This fact associated with the low inocula used in the experiments could explain the relatively low TP observed in only 5 out of 8 organs/tissue and a generally discrete or moderate inflammatory reaction in
8 organs/tissue examined. Only the genotype 20 , which is the more virulent, displayed severe inflammatory process. Such and other severe degenerative processes were never observed with the other clonal genotypes.

In previous studies (Lana et al., 1998; Laurent et al., 1997; Revollo et al., 1998), clonets 19 and 20 were plotted together, since they were very closely related and did not fall into distinct clusters when they were analyzed with 22 isozyme loci (Barnabé et al., 2000). However, this could be due to the fact that isozyme markers lack resolution for the lowest levels of phylo- 
genetic divergence. Although their clustering discreteness is not clear, these two clonal genotypes are reliably distinguished by one genetic marker, the isozyme locus 6 phosphogluconate dehydrogenase, which is homozygous for 19 and heterozygous for 20 (Tibayrenc et al., 1986). It is impossible to rule out the hypothesis that their medical and epidemiological relevance is different. In the present study, we have analyzed them separately. This made it possible to distinguish them for four important variables of virulence during the AP of the infection (PPP, MP, \%INF, and PAR), which demonstrated clearly the higher virulence of the genetic group 20 in comparison with genetic group 19. Although not always significant, histopathogical analysis also showed that mice infected with clones from this group showed more TP and inflammatory reaction in relation to all genotypes during the AP of infection. Genotypes 19 and 20, as well as "biodeme" III from Andrade and Magalhães (1997), displayed similar behavior and are classified as T. cruzi I (Satellite Meeting, Rio de Janeiro, Anon, 1999).

On the other hand, genetic groups 39 and 32, closely related and phylogenetically distant from the genetics groups 19 and 20, were different in six parameters, four of them related with virulence, specially PAR that was higher due to the Bug2149 c10 stock from the genotype 39. The lowest PPP and DMP of this genotype, not only in relation to genetic group 32 but also in relation to the other genotypes, indicate its high virulence. However the lowest PP and $\%+\mathrm{HC}$ during both phases of the infection suggest its higher susceptibility to host's immune response. These results corroborate those observed in mixed experimental infections in mice (Lana et al., 2000). In artificial mixtures involving either $19 / 20+39$ or $32+39$, the rates of successful reisolation were lower than for mixtures involving $19 / 20+32$ genotypes.

We have noticed an unexpected result in animals infected with stocks of the genotype 39 which did not show any TP AP of the infection, whereas data from the literature frequently show that detecting TP AP of the infection is easier, but not the contrary.

The other differences between 39 vs 32 were observed during the CP when the genotype 39 was the only one with TP, curiously not associated with inflammatory process, a fact already observed by other authors who have studied virulence and pathogenicity of different $T$. cruzi stocks in animal experiments (Anselmi et al., 1965; Lana et al., 1992). Maybe this contrasting result observed with stocks of the genotype 39 can be explained by the fact that this genotype is a hybrid clonet, harboring a combination of the genes of the two putative parental genotypes (Brisse et al., 2000).

Some previous results of Diego et al. (1998) concerning histopathological data were confirmed in the present work. These authors studied the AP of infection in Swiss mice infected with the 15 stocks of clonets 19,20 , and 39 that we have also analyzed. Similar results between these authors and us were observed: (i) for these three genotypes, the highest number of parasites and inflammatory lesions was observed in the skeletal muscles; (ii) the highest number of parasite in the skeletal muscles was observed with stocks of the genotype 39 during the CP. Contrary to Diego et al. (1998), we did not observe (i) parasites in lymphoid organs (liver and spleen) from mice infected with stocks of genotype 39; (ii) parasites and inflammatory lesions in the brain of mice infected with any genotype. Maybe the different inocula and lineage of mice used could explain these differences.

Considering the comparison between pairs of phylogenetically distant genotypes the higher number of significant differences was observed between 20 vs 39 (11 differences), and 19 vs 39 (9 differences). These results are overall consistent with the working hypothesis that biological differences are proportional to the evolutionary divergence among the genotypes, since closely related genotypes ( 19 vs $20-T$. cruzi I and 32 vs $39-T$. cruzi II) show fewer differences than distantly related groups (19 vs 39 and 20 vs 39 ).

However only four and six significant differences were recorded between 19 vs 32 , and 20 vs 32 , respectively, also phylogenetically distant. Differences between genotypes 19 vs 32 were recorded in parameters linked to virulence: higher $\% \mathrm{INF}, \mathrm{PAR}$, and $\%+\mathrm{HC}$ during the $\mathrm{CP}$ of the infection for the genotype 19. In the comparison of genotypes 20 vs 32 differences were also recorded in parameters of virulence, which show clearly the higher virulence of the genetic 20. This genotype shows higher PP, MP, PAR, $\% \mathrm{INF}, \%+\mathrm{HC} \mathrm{CP}$, and the lower PPP.

The working hypothesis of association between biological parameters and evolutionary divergence among natural clones is even more strongly supported by the result of the nonparametric Mantel test, which showed a highly significant correlation $\left(P=2.3 \times 10^{-3}\right)$ between biological differences and genetic distances among pairs of stocks. This shows that evolutionary divergence and biological differences do not evolve independently, and can be statistically predicted from each other to a large extent. This is actually another manifestation of the linkage disequilibrium generated by predominant clonal evolution in T. cruzi (Tibayrenc et al., 1986). A similar pattern has been observed in the bacterium Escherichia coli, which has a basically clonal population structure, although horizontal gene transfer is frequent in it (Miller and Hartl, 1986).

Finally, the present results corroborate those of other authors who have analyzed the same set of stocks for other parameters (Lana et al., 1998; Laurent et al., 1997; Revollo et al., 1998), several of which are of medical relevance (virulence in mice, transmissibility through the vector, infectivity of culture cells, in vitro drug sensitivity). Preliminary results showed that the in vivo sus- 
ceptibility of the same set of stocks to Benznidazole and Itraconazole (Toledo et al., 2000) is also consistent with this hypothesis. Moreover, experiments dealing with artificial mixture of stocks pertaining to different clonal genotypes in vectors (Pinto et al., 2000) and in mice (Lana et al., 2000) suggested in some cases an interaction between different stocks. All these results taken together emphasize the fact that it is crucial to take into account the phylogenetic diversity of $T$. cruzi natural clones in all applied studies dealing with diagnosis, drug and vaccine design, epidemiological surveys, and clinical diversity of Chagas' disease.

\section{Acknowledgments}

This work was supported by grants from FAPEMIG (Fundação de Amparo à Pesquisa de Minas Gerais), Brasil.

\section{References}

Andrade, S.G., 1976. Tentative for grouping different Trypanosoma cruzi strains in some types. Revista do Instituto de Medicina Tropical de São Paulo 18, 140-141.

Andrade, S.G., Magalhães, J.B., 1997. Biodemes and zymodemes of Trypanosoma cruzi strains: correlation with clinical data and experimental pathology. Revista da Sociedade Brasileira de Medicina Tropical 30, 27-35.

Andrade, S.G., Magalhães, J.B., Pontes, A.L., 1985. Evaluation of chemotherapy with Benznidazole and Nifurtimox in mice infected with Trypanosoma cruzi strains of different types. Bulletin of the World Health Organization 63, 721-726.

Andrade, S.G., Rassi, A., Magalhães, J.B., Filho, F., Luquetti, A.O., 1992. Specific chemotherapy of Chagas disease: a comparison between the response in patients and experimental animals inoculated with the same strains. Transactions of the Royal Society of Tropical Medicine and Hygiene 86, 624-626.

Andrade, V., Brodskyn, C., Andrade, S.G., 1983. Correlation between isoenzyme patterns and biological behavior of different strains of Trypanosoma cruzi. Transactions of the Royal Society of Tropical Medicine and Hygiene 77, 796-799.

Anon, 1999. International Symposium to commemorate the 90th anniversary of the discovery of Chagas disease. Recommmendations from a Satellite Meeting, Rio de Janeiro, Brazil, 16-4-1999. Memórias do Instituto Oswaldo Cruz. 94, (Suppl. I), pp. 429-432.

Anselmi, A., Pifano, F.C., Suarez, A., Dominguez, A., Vazquez, A.O., Anselmi, G., 1965. Experimental Schizotrypanum cruzi myocarditis. Correlation between histopathological and electrocardiographic findings in experimental Shags heart disease. American Heart Journal 72, 469-481.

Barbosa, A., 1985. Método imuno-histoquímico para a identificação de amastigotas de Trypanosoma cruzi em cortes histológicos de rotina. Revista do Instituto de Medicina Tropical de São Paulo 28, 91-96.

Barnabé, C., Brisse, S., Tibayrenc, M., 2000. Population structure and genetic typing of Trypanosoma cruzi, the agent of Chagas disease: a multilocus enzyme electrophoresis approach. Parasitology 150, 513-526.

Brener, Z., 1962. Therapeutic activity and criterion of cure on mice experimentally infected with Trypanosoma cruzi. Revista do Instituto de Medicina Tropical de São Paulo 4, 389-396.
Brener, Z., 1987. Pathogenesis and immunopathology of chronic Chagas disease. Memórias do Instituto Oswaldo Cruz 82, 205-212.

Brenière, S.F., Tibayrenc, M., Antezana, G., Pabon, J., Carrasco, R., Selaes, H., Desjeux, P., 1985. Résultats préliminaires en faveur d'une relation faible ou inexistante entre les formes cliniques de la maladie de Chagas et le souches isoenzymatiques de Trypanosoma cruzi. Comptes-Rendus de l' Academie des Sciences de Paris 300, 555-558.

Brisse, S., Barnabé, C., Tibayrenc, M., 1998. Trypanosoma cruzi: how many relevant phylogenetic subdivisions are there? Parasitology Today 14, 178-179.

Brisse, S., Barnabé, C., Tibayrenc, M., 2000. Identification of six Trypanosoma cruzi phylogenetic lineages by random amplified polymorphic DNA and multilocus enzyme electrophoresis. International Journal of Parasitology 30, 35-44.

Carneiro, M., Romanha, A.J., Chiari, E., 1991. Biological characterization of Trypanosoma cruzi strains from different zymodemes and schizodemes. Memórias do Instituto Oswaldo Cruz 86, 387-393.

Conares, W.J., 1980. Practical Non Parametric Statistics, second ed. John Wiley \& Sons, New York (Chapter 6).

Deane, M.P., Moriearty, P.L., Thomaz, N., 1984. Genes and other parasitic protozoa. In: Morel, C.M. (Ed.), Genes and Antigens of Parasites: A Laboratory Manual, second ed. Fundação Oswaldo Cruz, Rio de Janeiro, Brazil, pp. 11-21.

Dias, J.C.P., 1992. Epidemiology of Chagas disease. In: Wendel, S., Brener, Z., Camargo, M.E., Rassi, A. (Eds.), Chagas disease (American Trypanosomiasis): Its Impact on Transfusion and Clinical Medicine. ISBT BRAZIL-SBHH, São, Paulo, pp. 49-80.

Diego, J.A., Palau, M.T., Gamallo, C., Penin, P., 1998. Relationships between histopathological findings and phylogenetic divergence in Trypanosoma cruzi. Tropical Medicine and International Health 3, $222-233$.

Dvorak, J.A., 1984. The natural heterogeneity of Trypanosoma cruzi: Biological and medical implications. Journal of Cellular Biochemistry $24,357-371$.

Dvorak, J.A., Hartman, D.L., Miles, M.A., 1980. Trypanosoma cruzi: correlation of growth kinetics to zymodeme type in clones derived from various sources. Journal of Protozoology 27, 472-474.

Filardi, L.S., Brener, Z., 1987. Susceptibility and natural resistance of Trypanosoma cruzi strains to drugs used clinically in Chagas disease. Transactions of the Royal Society of Tropical Medicine and Hygiene 81, 755-759.

Garcia, E.S., Dvorak, J.A., 1982. Growth and development of two Trypanosoma cruzi clones in the arthropod Dipetalogaster maximus. The American Journal of Tropical Medicine and Hygiene 31, 259-262.

Jaccard, P., 1908. Nouvelles recherches sur la distribution florale. Bulletin de la Societé vaudoise de Sciences Naturelles 44, 223-270.

Lana, M., Chiari, E., Tafuri, W.L., 1992. Experimental Chagas disease in dogs. Memórias do Instituto Oswaldo Cruz 87, 59-71.

Lana, M., Pinto, A.S., Barnabé, C., Quesney, V., Noël, S., Tibayrenc, M., 1998. Trypanosoma cruzi: compared vectorial transmissibility of three major clonal genotypes by Triatoma infestans. Experimental Parasitology 89, 1-5.

Lana, M., Pinto, A.S., Bastrenta, B., Barnabé, C., Noël, S., Tibayrenc, M., 2000. Trypanosoma cruzi: Infectivity of clonal genotypes infections in acute and chronic phases in mice. Experimental Parasitology 96, 61-66.

Laurent, J.P., Barnabé, C., Quesney, V., Noël, S., Tibayrenc, M., 1997. Impact of clonal evolution on the biological diversity of Trypanosoma cruzi. Parasitology 114, 213-218.

Machado, C.A., Ayala, F.J., 2001. Nucleotide sequences provide evidence of genetic exchange among distantly related lineages of Trypanosoma cruzi. Proceedings of the National Academy of Science USA 98 (13), 7396-7401.

Mantel, N., 1967. The detection of disease clustering and a generalized regression approach. Cancer Research 27, 209-220. 
Marques De Araújo, S., Chiari, E., 1988. Caracterização biológica de clones das cepas Y, CL e MR de Trypanosoma cruzi em camundongos $\mathrm{C}_{3} \mathrm{H}$ isogênicos. Memórias do Instituto Oswaldo Cruz 83, 175-181.

Melo, R.C., Brener, Z., 1978. Tissue tropism of different Trypanosoma cruzi strains. Journal of Parasitology 64, 475-482.

Miles, M.A., Povoa, M.M., De Souza, A.A., Lainson, R., Shaw, J.J., Ketteridge, D.S., 1981a. Chagas disease in the Amazon Basin: II. The distribution of Trypanosoma cruzi zymodemes 1 and 3 Pará state, north Brazil. Transactions of the Royal Society of Tropical Medicine and Hygiene 75, 667-674.

Miles, M.A., Povoa, M., Prata, A., Cedillos, R.A., De Souza, A.A., Macedo, V., 1981b. Do radically dissimilar Trypanosoma cruzi strains (zymodemes) cause Venezuelan and Brazilian forms of Chagas disease? Lancet 8234, 1336-1340.

Miller, R.D., Hartl, D.L., 1986. Biotyping confirms a nearly clonal population structure in Escherichia coli. Evolution 40, $1-12$.

Pinto, A.S., Lana, M., Britto, C., Bastrenta, B., Tibayrenc, M., 2000. Experimental Trypanosoma cruzi biclonal infection in Triatoma infestans: detection of distinct clonal genotypes using kinetoplast DNA probes. International Journal for Parasitology 30, 843-848.

Rassi, A., Luquetti, A.O., 1992. Therapy of Chagas disease. In: Wendel, S., Brener, Z., Camargo, M.E., Rassi, A. (Eds.), Chagas Disease (American Trypanosomiasis): Its Impact on Transfusion and Clinical Medicine. ISBT BRAZIL'92, São Paulo, pp. 237-256.

Revollo, S., Oury, B., Laurent, J.P., Barnabé, C., Quesney, V., Carriére, V., Noël, S., Tibayrenc, M., 1998. Trypanosoma cruzi: impact of clonal evolution of the parasite on its biological and medical properties. Experimental Parasitology 89, 30-39.

Snedecor, G.W., Cochran, W.G., 1989. Statistical Methods, eighth ed. Iowa State University Press, Ames, 503pp.

Souto, R.P., Fernandes, O., Macedo, A.M., Campbell, D.A., Zingales, B., 1996. DNA markers define two major phylogenetic lineages of Trypanosoma cruzi. Molecular and Biochemical Parasitology 83, $141-152$.

Tibayrenc, M., 1995. Population genetics of parasitic protozoa and other microoganisms. Advanced in Parasitlogy 36, 47-115.

Tibayrenc, M., Ayala, F.J., 1988. Isozyme variability in Trypanosoma cruzi, the agent of Chagas disease: genetical, taxonomical, and epidemiological significance. Evolution 42, 277-292.

Tibayrenc, M., Brenière, S.F., 1988. Trypanosoma cruzi: major clones rather than principal zymodemes. Memórias do Instituto Oswaldo Cruz 83, 249-255.

Tibayrenc, M., Neubauer, K., Barnabé, C., Guerrini, F., Skarecky, D., Ayala, F.J., 1993. Genetic characterization of six parasitic protozoa: parity between random-primer DNA typing and multilocus enzyme electrophoresis. Proceedings of the National Academy of Sciences of the USA 90, 1335-1339.

Tibayrenc, M., Ward, P., Moya, A., Ayala, F.J., 1986. Natural populations of Trypanosoma cruzi, the agent of Chagas disease, have a complex multiclonal structure. Proceeding of the National Academy of Sciences of the USA 83, 115-119.

Toledo, M.J.O., Lana, M., Bahia, M.T., Carneiro, C.M., Alves, C.F., Martins, H.R., Tibayrenc, M., Tafuri, W.L., 2000. Evaluation of chemotherapy with Benznidazole and Itraconazole in mice infected with different Trypanosoma cruzi clonal genotypes. Memórias do Instituto Oswaldo Cruz 95 (Suppl. II), 314. 